\title{
The Chemical Evolution of the Universe
}

\author{
Antoinette Songaila \& Lennox L. Cowie \\ Institute for Astronomy, 2680 Woodlawn Drive, Honolulu, HI 96822, \\ USA
}

\begin{abstract}
.
We still know relatively little about the local metal density outside of galaxies and clusters, but at high redshifts $(z \sim 3)$ the advent of highly efficient high-resolution spectrographs on the new 8-10m telescopes has revolutionized the study of quasar absorption lines. This allows us to track the bulk of the baryons in the Universe, which are in highly observable form at this redshift, and to determine the metal density of the gas that contains most of the baryonic material. One of the more surprising results that has emerged is how uniformly the intergalactic gas at redshifts $2-4$ is pervaded by metals, and we discuss current limits on such metal contamination of the low density gas. These measurements will be useful in understanding and calibrating feedback processes in early generations of small galaxies. Futurè work will track the metal distribution at higher redshift using bright $z=5$ SDSS quasars.
\end{abstract}

\section{Introduction}

We are currently in the paradoxical situation in which we know much more about the metal content of the bulk of the baryons at $z=3$ than we do about the equivalent situation in the low- $z$ universe. Locally, we have a baryon density of roughly $\rho=2 \times 10^{-32} \mathrm{~g} \mathrm{~cm}^{-3}$ in galaxies carrying a metal density of roughly $4 \times 10^{-34} \mathrm{~g} \mathrm{~cm}^{-3}$ for $H_{0}=50 \mathrm{~km} \mathrm{~s}^{-1} \mathrm{Mpc}^{-1}$ (e.g., Cowie 1988; Fukugita et al. 1998), but much of the baryon content is unseen and probably lies in modestly hot intergalactic gas (Mushotzky, this volume) about whose metal content we know nothing. In contrast, at $z=3$ the bulk of the baryons appear to lie in warm ionized gas, which is accessible to direct measurement. The presence and distribution of heavy elements in the intergalactic medium (IGM) at high redshift is turning out to be a crucial discriminant of models of structure formation in the early Universe. This talk will focus on our understanding of the metals at these high redshifts.

The physical picture of the evolution of the IGM that has been emerging both from numerical simulations and from other approximation techniques (Cen et al. 1994; Zhang, Anninos, \& Norman 1995; Hernquist et al. 1996; Bi \& Davidsen 1997; Hui, Gnedin, \& Zhang 1997; Gnedin \& Hui 1998) explains the Lyman alpha forest seen in quasar absorption line spectra in the context of the gravitational growth of structure in the intergalactic gas. In particular, the absorbers that give rise to low column density lines $\left(N(\mathrm{H} \mathrm{I})<10^{15} \mathrm{~cm}^{-2}\right)$ at 


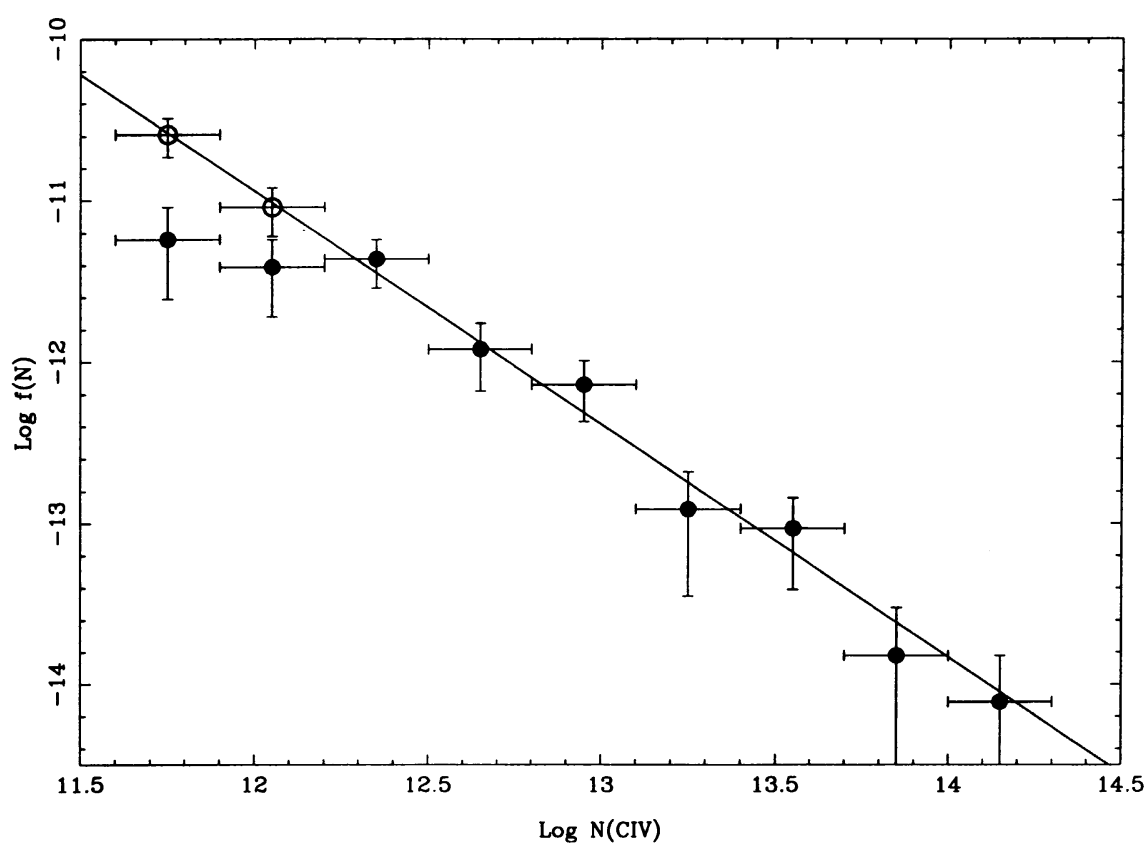

Figure 1. Column density distribution of C IV absorbers in Q1422+231 where $f(\mathrm{~N})$ is the number of systems per column density interval per unit redshift path. The data (solid points) have been grouped into bins of width 0.3 in $\log N$ (C IV) for display purposes only. The best-fit power law with index $\alpha=1.44 \pm 0.05$ is indicated by the solid line. The two lowest bins show signs of incompleteness as lines with large $b$-values and low column density may not have been identified. Correction factors were determined by simulations, and corrected points are shown with open circles.

redshifts $z \sim 3$ arise from the gas in large unvirialized objects with densities close to the cosmic mean. The widths of lines are set by the Hubble expansion across their width (Weinberg et al. 1997), as well as by thermal or turbulent velocities, and in this limit there is a rough linear relation between the optical depth and the baryon overdensity (Croft et al. 1998), so that the lowest column density lines provide information about the intergalactic medium at or below the average cosmic density, whereas higher column density lines are produced in more overdense regions.

In parallel with this theoretical understanding, the HIRES spectrograph on Keck I has provided a breakthrough in observations of the IGM, giving us exquisite high resolution spectra of $z=2-4$ quasars, which allowed us to study, among other things, the extremely weak Lyman alpha forest lines that arise in IGM material close to the mean density. One of the most important discoveries was the detection of widespread contamination by metals in the IGM (Cowie et al 1995; Tytler et al. 1995). Using the straightforward methodology of identifying metal-line systems corresponding to weak Lyman forest systems, we 
showed (Songaila \& Cowie 1996) that C IV can be found in $75 \%$ of clouds with $N(\mathrm{H} \mathrm{I})>3 \times 10^{14} \mathrm{~cm}^{-2}$, which corresponds to an overdensity of about $\delta \sim 5$ in the IGM at $z \sim 3$. Such direct measurements have been carried to their current limits by Ellison et al. (2000), who used a HIRES spectrum of the extremely bright lensed quasar Q1422+231 to measure C IV lines to a limiting sensitivity of $\log N(\mathrm{C} \mathrm{IV})=11.75$. To this level, the number of metal-line systems continues to rise smoothly as a -1.4 power law, paralleling the slope of the $\mathrm{H}$ I clouds in the Ly $\alpha$ forest (Figure 1). This result already implies that metals permeate much of the intergalactic gas, an extremely surprising result that has yet to be fully understood.

One of the most important outstanding questions is how the metals are distributed into the intergalactic gas, and in particular, whether metals reside in the average density IGM or are restricted to overdense regions. If forest clouds are associated with galaxies, large radii $\left(\sim 200 \mathrm{~h}^{-1} \mathrm{kpc}\right)$ are required to account for the number density of $N(\mathrm{H} \mathrm{I})>10^{15} \mathrm{~cm}^{-2}$ clouds at $z=3$ (e.g., Steinmetz 1997), and it appears improbable that star formation in the galaxy itself could contaminate this entire region (Aguirre et al. 2000a, b; Theuns, Mo, $\&$ Schaye 2000; Efstathiou 2000). The remaining possibilities are that the metals are being produced by star formation in the clouds themselves (e.g., Madau \& Shull 1996), perhaps at an early stage in the galaxy formation process, or that they are formed in an even earlier stage of metal production at much higher redshift that has uniformly enhanced the intergalactic medium (e.g., Gnedin \& Ostriker 1997). Since this last process presumably would occur on a sub-galactic scale, we might expect it to produce a more uniform metallicity at the scale of the clouds; in contrast, we would expect in situ enrichment to depend heavily on the properties of individual clouds and perhaps to be strongest in higher density clouds. Understanding these questions is critical to modelling the earliest generation of small galaxies and also to understanding how they expelled the metals into the more general IGM. Indeed, while the agreement between current models and observations is extremely good (Haehnelt, Steinmetz, \& Rauch 1996; Davé et al. 1997; Hellsten et al. 1997; Machacek et al. 1998) and suggests that they are fundamentally on the right track, it remains possible that very energetic processes from early galaxy formation associated with the star formation that produced the observed metals could result in a multi-phase intergalactic gas with considerably different properties.

\section{Metals in the Low Density IGM}

While moderate density $\left(N(\mathrm{H} \mathrm{I})>3 \times 10^{14} \mathrm{~cm}^{-2}\right)$ clouds are now well characterized as having a spread of less than an order of magnitude in both volume density and metallicity, a carbon abundance of very approximately $10^{-2.5}$ of solar and $\mathrm{Si} / \mathrm{C}$ about three times solar (Songaila \& Cowie 1996; Davé et al. 1998), the measurement of the abundance of heavy elements in the low density IGM is not so straightforward, and contradictory results exist.

There has been wide speculation that metallicities may fall rapidly at lower column densities since galactic enrichment and the merger expulsion and supernova generated wind mechanisms responsible for distributing the heavy elements back into the intergalactic gas should be more efficient in higher overdensity 


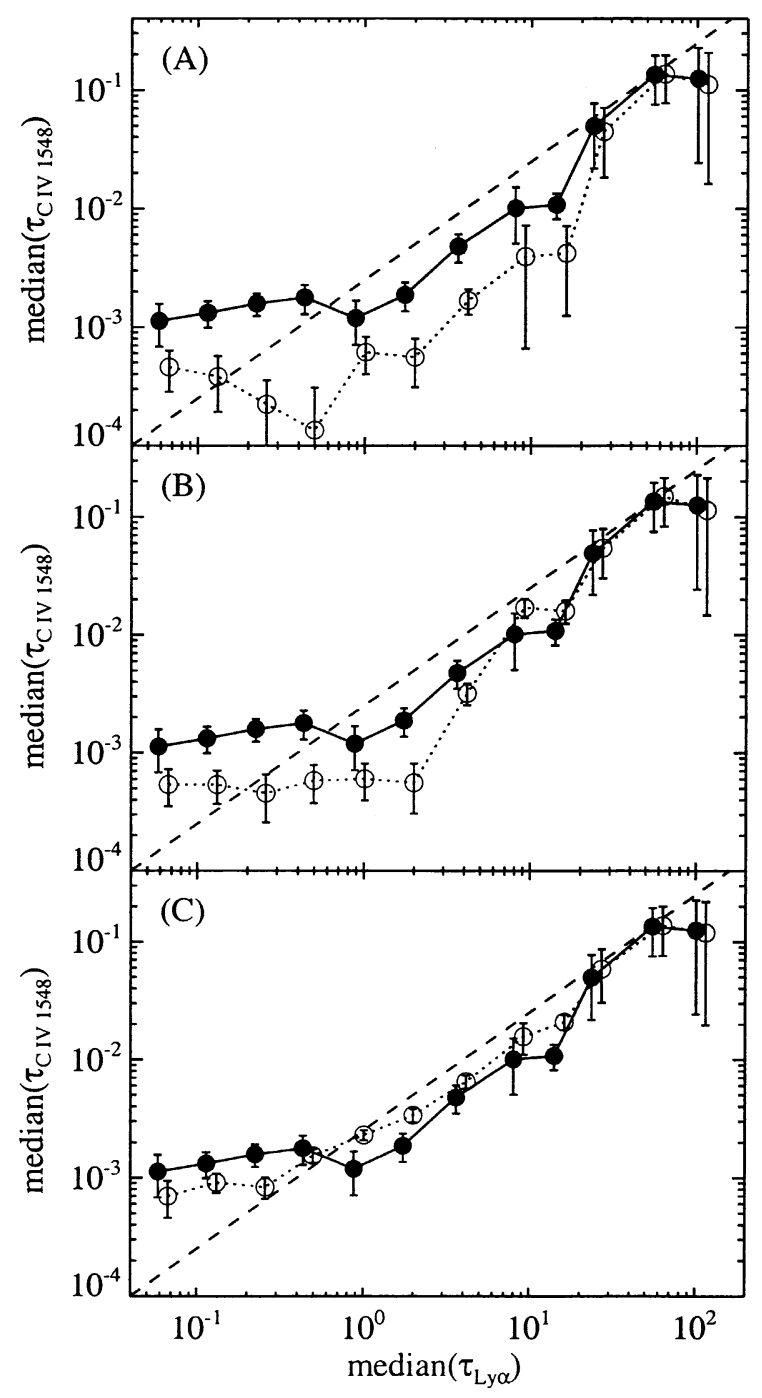

Figure 2. Results from the optical depth analysis of Q1422+231 (solid points) compared with three synthetic spectra (open circles in all three cases). Top panel: the synthetic spectrum is enriched solely with detected C IV systems. Middle panel: In addition, $\log N(\mathrm{C}$ IV $)=12.0$ is included in all Ly $\alpha$ clouds with $\log N(\mathrm{H} \mathrm{I})>14.5$, representing the maximum amount of metals that could be 'hidden' below the detection limit in strong absorbers. Bottom panel: Supplementary C IV is now added in all weak $(\log N(\mathrm{H} \mathrm{I})<14.5)$ Ly $\alpha$ lines with $\log \mathrm{C} \mathrm{IV} / \mathrm{H} \mathrm{I}=$ -2.6 . Clearly, more C IV is present than currently identified directly and these optical depth results show that the data are consistent with a significant amount of C IV in low column density clouds. 
regions. In particular, early pre-enrichment models (Gnedin \& Ostriker 1997; Gnedin 1998) in which the metals are formed in sub-galactic clumps at $z>5$ predict that at $z \sim 3$ the metallicity should begin to fall rapidly below an overdensity of about $\delta \sim 10$, close to the lowest column densities at which metallicity measurements have been made. There is therefore a strong motivation to push the metal determinations to as low an overdensity (column density) as possible to investigate whether this very robust prediction is correct.

Previous attempts (Norris, Peterson, \& Hartwick 1983; Lu 1991; Tytler \& Fan 1994; Lu et al. 1998) to measure metallicity at lower densities have generally focussed on coadding metal lines in some way to improve the signal-to-noise ratio. Ellison et al. (2000) used this technique on a combined spectrum of Q1422+231 and APM $08279+5255$, producing a stacked spectrum with S/N of 1250 , with no C IV being detected. However, it is likely that this and previous attempts to detect weak C IV in this way are beset by systematic problems. In Ellison et al. we identified a number of these, the most important being random velocity offsets between $\mathrm{C} I V$ and $\mathrm{H} \mathrm{I}$. We have recently evolved a very different technique of directly correlating metal line and $\mathrm{H}$ I optical depths (Songaila 1998; Cowie \& Songaila 1998; Ellison et al. 2000) which has the advantage of being highly sensitive, of avoiding subjective Voigt profile fitting, and of allowing objective assessment of noise levels, including systematics. We have shown, using this technique, that $\mathrm{C} \mathrm{IV/H} \mathrm{I} \mathrm{remains} \mathrm{roughly} \mathrm{constant} \mathrm{down} \mathrm{to} \mathrm{at} \mathrm{least}$ $N(\mathrm{H} \mathrm{I}) \sim 10^{14} \mathrm{~cm}^{-2}$, corresponding to $\delta \sim 1$, or "average" IGM gas. This is illustrated in Figure 2, which shows detailed comparisons of the optical depth distribution of our observed spectrum of Q1422+231 with synthetic spectra (Ellison et al. 2000). In the top panel, the synthetic forest spectrum is enriched only with directly detected C IV systems, and plainly fails to reproduce the lower optical depth points. The location of the extra required absorption is tested in the other two panels. In the middle panel, the synthetic spectrum is enriched with "hidden" C IV at the $\log N(\mathrm{C}$ IV $)=12.0$ level only in the stronger clouds $[\log N(\mathrm{H} \mathrm{I})>14.5]$. Agreement with the observed distribution is reached (bottom panel) only when extra C IV is also added in weak clouds, with $\log \mathrm{C} I V / \mathrm{H} \mathrm{I}=-2.6$. Clearly, more $\mathrm{C} \mathrm{IV}$ is present than is currently detected directly and, more crucially, a significant amount of this must be in the low column density clouds which correspond to low density intergalactic gas. This is an extremely powerful technique, capable of reaching the lowest densities required with additional data of the quality of Q1422+231, which is still our current highest $\mathrm{S} / \mathrm{N}$ spectrum. Confirmation of near-constant metallicity down to these very low densities, typical of the voids, would be a very important result. It would mean that the earliest generations of galaxies were extraordinarily efficient at reintroducing metals, and presumably energy as well, into the IGM, with important implications for modelling the early IGM, which may have more complex structure and dynamics than the current models are taking into account.

\section{Metals at $z=3$}

We now have enough metal line data to get a direct handle on the metal density of the Universe at $z \sim 3$, independent of other assumptions such as the value of 

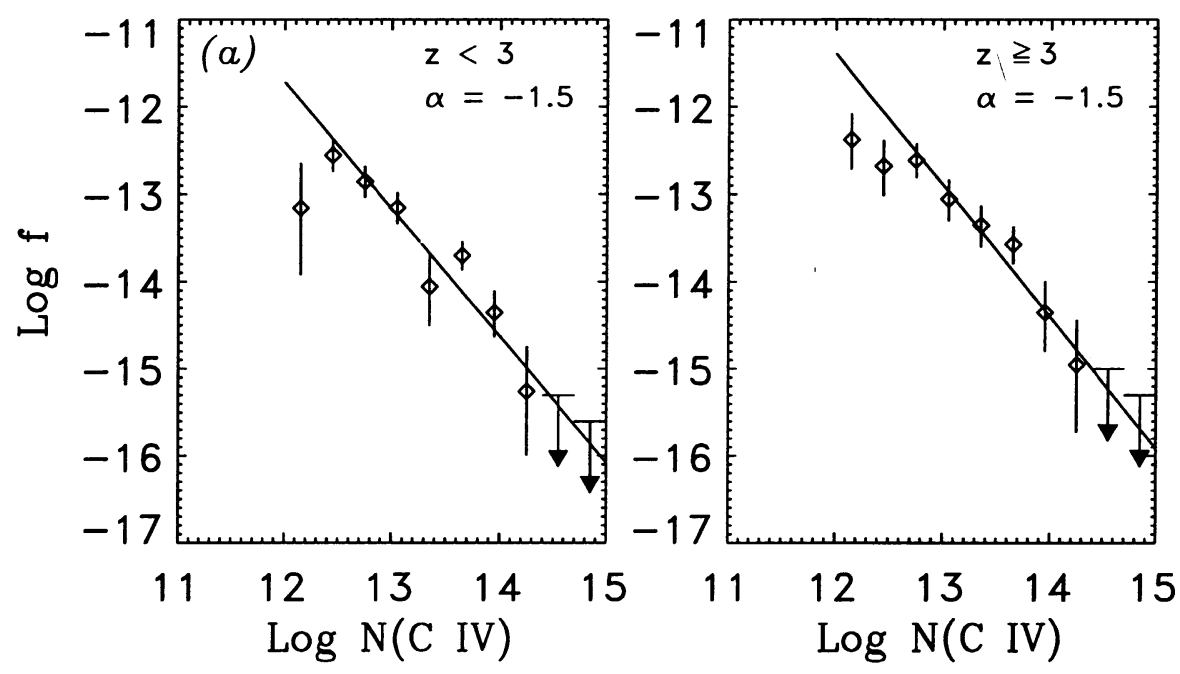

Figure 3. C IV column density distribution functions for $z<3$ and $z \geq 3$ for a total of 81 absorption line systems in seven lines of sight toward distant quasars. The bin size is $10^{0.3} \mathrm{~N} \mathrm{~cm}^{-2}$. Error bars are $\pm 1 \sigma$, based on the number of systems in each bin. The solid lines show the best fit power law with index $\alpha=-1.5$, fitted to data above $6 \times 10^{12} \mathrm{~cm}^{-2}$ where the data is substantially complete.

$\Omega_{b}$, just by directly integrating the C IV and Si IV column densities, and this measurement was carried out by Songaila (1998). Fig. 3 shows the C IV column density distribution, $f(N)$, which is the number per unit column density per unit redshift path, split by redshift interval, approx $2-3$ and $3-3.5$ and a similar figure for silicon can be found in Songaila (1998). The total sample is 81 C IV systems and $35 \mathrm{Si}$ IV systems, all lying outside the Ly $\alpha$ forest, drawn from 7 QSO lines of sight, excluding systems close to the quasar. Power law fits over the column densities $\log N(\mathrm{C}$ IV $)=12-14.3$, and $\log N(\mathrm{Si}$ IV $)=11.70-13.9$ give power law indices of -1.5 for both low and high redshift $\mathrm{C}$ IV and -1.8 for low-redshift Si IV and -2 for high redshift Si IV. (These power laws are fitted to the regions where the data are substantially complete.) As one would expect, the C IV data show the same power law slope as $\mathrm{H} \mathrm{I}$, reflecting the fact that the $\mathrm{C} \mathrm{IV/H} \mathrm{I} \mathrm{value} \mathrm{is} \mathrm{nearly} \mathrm{constant} \mathrm{with} \mathrm{redshift.}$

The density of C IV and Si IV can now be directly integrated from the C IV and Si IV distributions. For the $z>3$ systems this gives the values $\Omega_{\mathrm{C} \text { IV }}=$ $(1.2 \pm 0.3) \times 10^{-8} h^{-1}\left[1+2 q_{0} z\right]^{1 / 2}$ and $\Omega_{\mathrm{Si}} \mathrm{IV}=(4.3 \pm 1.6) \times 10^{-9} h^{-1}\left[1+2 q_{0} z\right]^{1 / 2}$. The errors are $1 \sigma$ (statistical error only). We have restricted ourselves to $N(\mathrm{C}$ IV $)<3 \times 10^{14} \mathrm{~cm}^{-2}$ because higher column density systems are much 


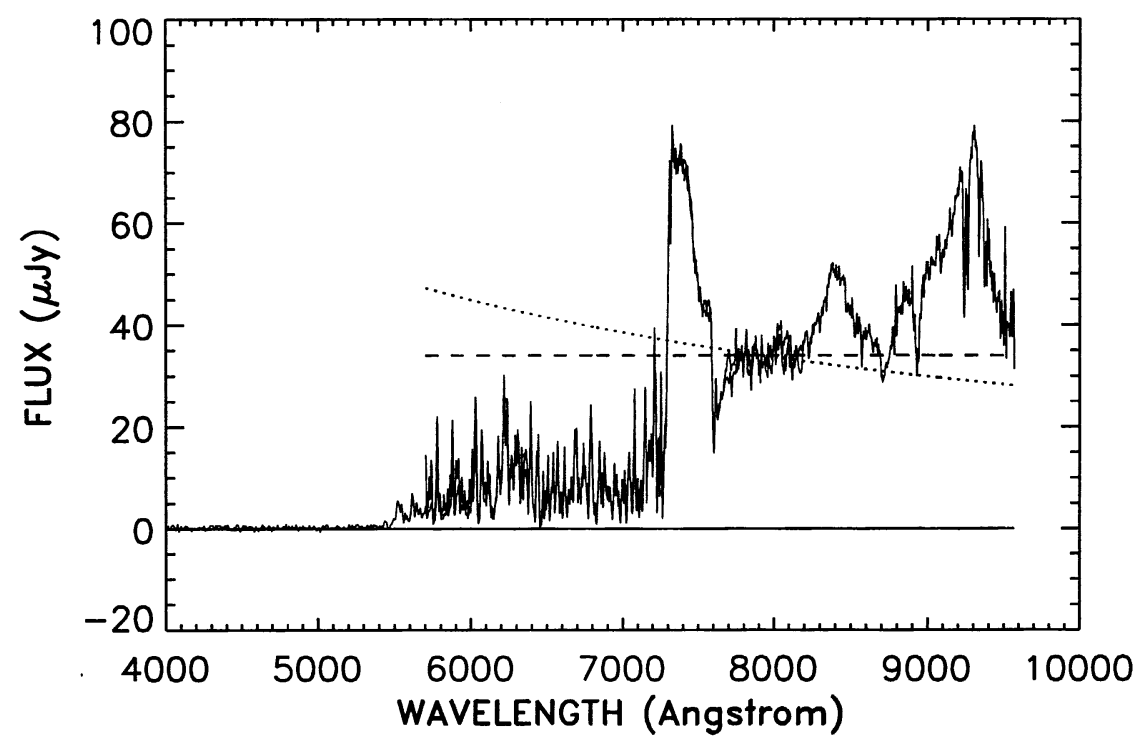

Figure 4. A spectrum of the $z=5$ quasar SDSS J0338+0021, shown in $\mu \mathrm{Jy}$ versus wavelength. The dashed and dotted lines show $\nu^{0}$ and $\nu^{1}$ continuum fits, normalized to the region $7700-8000 \AA$, which lies redward of the atmospheric A band but blueward of the Si IV emission line. Irrespective of the extrapolation, the Lyman break is extremely strong and the forest highly blended.

rarer and require a longer redshift path to determine the number density. The contribution to both C IV and Si IV converges at the low column density end, so that weaker than observed systems will not contribute much unless there is a radical upturn in the distribution at the low end. One now has to correct for ionization level. As already shown, the dominant ionization stage is triply ionized. C II/C IV is less than 0.1 , and the implied high ionization then means that Si III is less than Si IV and C III is less than C IV, and even with forest contamination, Si III is comparable to Si IV. N V/C IV is seen to be small in the few systems we have. So our best guess is $\Omega_{\mathrm{C}} \sim 2 \Omega_{\mathrm{C}}$ IV and the same for silicon. To get the overall metal density in a crude fashion, we scale the $\alpha$ process elements $(\mathrm{O}, \mathrm{Ne}, \mathrm{Si}, \mathrm{Mg}, \mathrm{S})$ with $\mathrm{Si}$ and the Fe-coproduction elements $(\mathrm{C}, \mathrm{N}, \mathrm{Fe})$ with $\mathrm{C}$ to give the metal density $\Omega_{\text {metals }}=(3.3 \pm 0.8) \times 10^{-7} h_{65}^{-1}\left(1+2 q_{\circ} z\right)^{0.5}$.

Does this check with what we know about the high redshift Universe? In particular, the absence of an H I Gunn-Peterson effect at the highest redshifts means, of course, that the hydrogen is highly ionized to $z=5$. Is this consistent with what we see of the effects of metal production, presumably at these same redshifts? Can the stars that form the metals ionize the intergalactic gas? The production of metals approximately calibrates the production of ionizing photons. The energy in ionizing photons/baryon is as given, just the energy 
from nucleosynthesis. This works out that $Z \sim 10^{-5}$ gives one ionizing photon/baryon, so that the value we see (a minimum) comes out to be about 2 photons per baryon, just enough to ionize the Universe.

\section{Metals at Higher Redshift}

An alternate way to explore these issues is to measure the metal distributions at yet higher redshifts. It is becoming practical to obtain substantial samples in the redshift range, $z=4-6$ as the Sloan digital sky survey is now yielding quasars (Fan et al. 1999, 2000, 2001; Zheng et al. 2000) (Fig. 4) which are bright enough that we can obtain high quality high resolution spectra. The ESI spectrograph on the Keck II $10 \mathrm{~m}$ telescope provides highly efficient coverage of the whole spectrum $(0.39-1.1 \mu \mathrm{m})$, permitting a systematic study of the properties of the gas and metals at these redshifts.

These observations will be extremely useful for the metal studies, since the rise in the overall column densities means that, while we cannot reach quite as sensitive limits in the metal lines because the quasars are fainter and we are working in the more difficult red portion of the spectrum, we can nevertheless probe to comparable overdensities in the gas, again allowing us to test how the metals are distributed in near-average density intergalactic gas. Hopefully, in the next year or two we will begin to see metal estimates similar to those at $z=2-4$ at these higher redshifts, and will determine if there is evolution in the the higher redshift range.

\section{Acknowledgements}

We wish to thank Sara Ellison and Max Pettini for much insight into the optical depth method. This work was supported in part by NSF grant AST96-17216.

\section{References}

Aguirre, A., Hernquist, L., Weinberg, D., Katz, N., \& Gardner, J. 2000a, ApJ, in press (astro-ph/0006345)

Aguirre, A., Hernquist, L., Katz, N., Gardner, J., \& Weinberg, D. 2000b, ApJ, in press (astro-ph/0006346)

Bi, H. G., \& Davidsen, A. 1997, ApJ, 479, 523

Cen, R. Y., Miralda-Escudé, J., Ostriker, J. P. O., \& Rauch, M. 1994, ApJ, 437, L9

Cowie, L. L. 1998, in The Post-Recombination Universe, ed. N. Kaiser \& A. Lasenby (Kluwer: Dordrecht), 1

Cowie, L. L., \& Songaila, A. 1998, Nature, 394, 44

Cowie, L. L., Songaila, A., Kim, T.-S., \& Hu, E. M. 1995, AJ, 109, 1522

Croft, R. A. C., Weinberg, D. H., Katz, N, \& Hernquist, L. 1998, ApJ, 495, 44

Davé, R., Hernquist, L., Wienberg, D. H., \& Katz, N. 1997, ApJ, 477, 21 
Davé, R., Hellsten, U., Hernquist, L., Katz, N., \& Weinberg, D. H. 1998, ApJ, 509,661

Efstathiou, G. 2000, MNRAS, 317, 697

Ellison, S. L., Songaila, A., Schaye, J., \& Pettini, M. 2000, AJ, 120, 1175

Fan, X., et al. 1999, AJ, 118, 1

Fan, X. et al. 2000, AJ, 119, 1

Fan, X., Strauss, M. A., Richards, G. T., Newman, J. A., Becker, R. H. et al. 2001, AJ, in press (astro-ph/0008122)

Fukugita, M., Hogan, C. J., \& Peebles, P. J. E. 1998, ApJ, 503, 518

Gnedin, N. Y., \& Ostriker, J. P. O. 1997, ApJ, 486, 581

Gnedin, N. Y. 1998, MNRAS, 294, 407

Gnedin, N. Y., \& Hui, L. 1998, MNRAS, 296, 44

Haehnelt, M. G., Steinmetz, M., \& Rauch, M. 1996, ApJ, 465, L95

Hellsten, U., et al. 1997, ApJ, 487, 482

Hernquist, L., Katz, N., Weinberg, D. H., \& Miralda-Escudé, J. 1996, ApJ, 457, L5

Hui, L., Gnedin, N. Y., \& Zhang, Y. 1997, ApJ, 486, 599

Lu, L. 1991, ApJ, 379, 99

Lu, L., Sargent, W. L. W., Barlow, T. A., \& Rauch, M. 1998, preprint [astro$\mathrm{ph} / 9802189]$

Madau, P., \& Shull, J. M. 1996, ApJ, 457, 551

Machacek, M., Bryan, G., Anninos, P., Meiksin, A., \& Norman, M. 1998, Proc. of 6th International Symposium, PASCOS 98, ed. P. Nath (World Scientific), 43

Norris, J., Peterson, B. A., \& Hartwick, F. D. A. 1983, ApJ, 273, 450

Songaila, A., \& Cowie, L. L. 1996, AJ, 112, 335

Songaila, A., 1998, AJ, 115, 2184

Steinmetz, M. 1997, Proceedings of the 13th IAP Colloquium: Structure and Evolution of the IGM from QSO Absorption Line Systems, ed. P. Petitjean \& S. Charlot, 281

Theuns, T., Mo, H. J., \& Schaye, J. 2000, MNRAS, in press (astro-ph/0006065)

Tytler, D., \& Fan, X.-M. 1994, ApJ, 424, L87

Tytler, D., et al. 1995, in QSO Absorption Lines, ESO Astrophysics Symposia, ed. G. Meylan (Heidelberg: Springer), 289

Weinberg, D. H., Hernquist, L., Katz, N., Croft, R., \& Miralda-Escudé, J. 1997, Proceedings of the 13th IAP Colloquium, Structure and Evolution of the Intergalactic Medium from QSO Absorption Lines, ed. P. Petitjean \& S. Charlot (Paris: Nouvelles Frontières), 133

Zhang, Y., Anninos, P., \& Norman, M. L. 1995, ApJ, 453, L57

Zheng, W., Tsvetanov, Z. I., Schneider, D. P., Fan, X., Becker, R. H., et al. 2000, AJ, in press (astro-ph/0005247) 